\title{
2018 Athanasiou ABME Student Awards
}

The Kyriacos and Kiley Athanasiou Endowment was established in 2017 within the Biomedical Engineering Society (BMES) to promote biomedical engineering scholarship and honor the contributions of Dr. Kyriacos Athanasiou to the field. One goal of the endowment is to recognize excellence in future leaders of biomedical engineering by awarding the top papers published in the Annals of Biomedical Engineering (ABME) by graduate students and post-doctoral scholars. The first Athanasiou ABME Student Award Session was held at the 2018 BMES Annual Meeting. Six awardees were selected, and presented their papers at the session.

The Athanasiou ABME Student Awards were selected from all papers with graduate student or postdoctoral scholar first authors that were accepted and published online between July 1, 2017 and June 30, 2018. There were a total of 86 papers that met the criteria, with 70 written by graduate students and 16 written by post-doctoral scholars. The number of downloads, impact, and quality of the papers were used to select the awardees. Each deputy editor-inchief selected 6 finalists, from which the editor-in-chief selected the 6 award winners. The first author from each paper was contacted to notify them and ask them to present their work at the award session. The 2018 awardees were geographically diverse, with two from the United States, two from the United Kingdom, one from the Netherlands, and one from China. Five awardees attended the session in person to present, and one sent a pre-recorded video presentation (Fig. 1). The papers covered a wide range of topics including injury biomechanics, medical robotics, and orthopedics.

Horeman et al. evaluated different imaging techniques that could be employed in a steerable needle to treat cartilage defects. ${ }^{3}$ Injectable hydrogels have recently been developed to treat these defects, but the hydrogel has to be injected at the site of the defect, requiring local tissue characterization during the procedure. This characterization can be accomplished with small imaging systems, such as intravascular ultrasound or optical coherence tomography. The results of the study showed that optical coherence tomography provided the most accurate characterization compared to the gold standard of micro-computed tomography. This topic was especially fitting for an
Athanasiou Award given that most of Dr. Athanasiou's research has focused on cartilage.

Singh et al. compared the technical performance and brain function of surgeons during conventional laparoscopy and robotic surgery. ${ }^{6}$ Surgeons performed a suturing task under a time constraint, and the results showed improved technical performance and greater prefrontal cortex activation during robotic surgery. This study suggests that robotic surgery could both improve technical performance and reduce the mental load for surgeons under high workload conditions.

Horvath et al. compared different fixation strategies for coupling a cardiac compression sleeve to the heart. ${ }^{4}$ A soft robotic cardiac assist device can be used to augment ventricular function in patients with endstage heart failure, but efficient coupling of the device to the surface of the heart presents a challenge. This study showed that a mesh-based sleeve allowed to biologically integrate with the surface of the heart provided better coupling and may improve heart function.

Liu et al. proposed an augmented-reality based navigation system to assist surgeons during hip resurfacing. ${ }^{5}$ The system is employed with a headset that surgeons use during the procedure to reduce implantation error of reconstructed articular surfaces. The system was tested by drilling guide holes in a femur phantom, and showed an acceptable level of accuracy for those tests.

Campolettano et al. evaluated head and neck injury associated with drone impacts. ${ }^{1}$ They tested three commercially available drones in both live flight and drop-style impacts to a Hybrid III test dummy head. The results showed that injury risk increases with increasing drone mass, and that larger models are not safe to fly over people in the case of an impact. This study could help inform drone flight regulations as they increase in popularity for the general public and commercial use.

Chan et al. compared different conventional materials in a scoliosis brace to a shape memory alloy. ${ }^{2}$ The braces were mechanically characterized and clinically tested. The shape memory alloy provided more continuous and controllable corrective forces, as well as better thoracic curvature correction in patient testing. These results suggest that shape memory alloys show promise for use in non-invasive orthopedic treatments. 


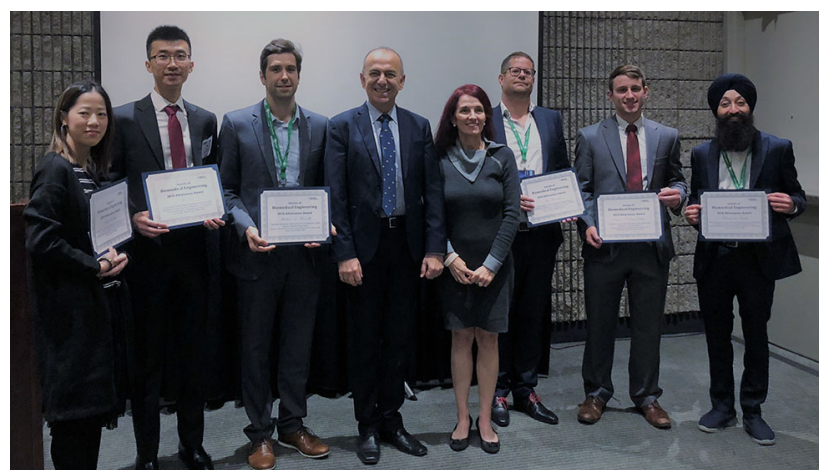

FIGURE 1. 2018 Athanasiou ABME Student Award Session. Pictured left to right: Anne Hon (representing Wing-Yu Chan), He Liu, Markus Horvath, Kyriacos Athanasiou, Kiley Athanasiou, Tim Horeman, Eamon Campolettano, and Harsimrat Singh.

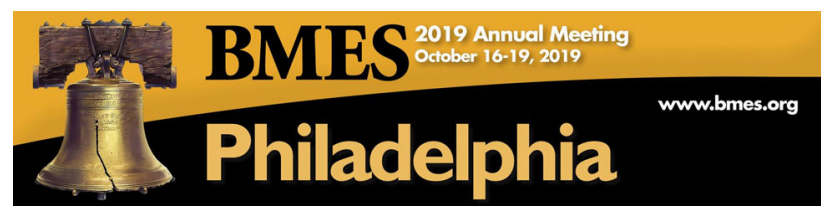

FIGURE 2. Join us for the next Athanasiou Award Session at the 2019 BMES Annual Meeting in Philadelphia.

The next Athanasiou ABME Student Award Session will be held at the 2019 BMES Annual Meeting in Philadelphia (Fig. 2). Papers with graduate student or post-doctoral scholar first authors that were accepted and published online between April 1, 2018 and March 31, 2019 will be considered for the award. Awardees will be notified by May 1, 2019. Each awardee will receive a $\$ 500$ honorarium and free meeting registration, and will be asked to present their paper at the award session. We look forward to another excellent session showcasing the best graduate student and postdoctoral scholar papers published in ABME.

\section{REFERENCES}

${ }^{1}$ Campolettano, E. T., M. L. Bland, R. A. Gellner, D. W. Sproule, B. Rowson, A. M. Tyson, S. M. Duma, and S. Rowson. Ranges of injury risk associated with impact from unmanned aircraft systems. Ann. Biomed. Eng. 45(12):27332741, 2017.

${ }^{2}$ Chan, W.-Y., J. Yip, K.-L. Yick, S.-P. Ng, L. Lu, K. M.-C. Cheung, K. Y.-H. Kwan, J. P.-Y. Cheung, K. W.-K. Yeung, and C.-Y. Tse. Mechanical and clinical evaluation of a shape memory alloy and conventional struts in a flexible scoliotic brace. Ann. Biomed. Eng. 46:1-12, 2018.

${ }^{3}$ Horeman, T., E. Buiter, B. Pouran, M. Stijntjes, J. Dankelman, and G. Tuijthof. In-vitro detection of small isolated cartilage defects: intravascular ultrasound vs. optical coherence tomography. Ann. Biomed. Eng. 46(11):17451755, 2018.

${ }^{4}$ Horvath, M. A., C. E. Varela, E. B. Dolan, W. Whyte, D. S. Monahan, C. J. Payne, I. A. Wamala, N. V. Vasilyev, F. A. Pigula, and D. J. Mooney. Towards alternative approaches for coupling of a soft robotic sleeve to the heart. Ann. Biomed. Eng. 46:1-14, 2018.

${ }^{5}$ Liu, H., E. Auvinet, J. Giles, and F. R. y Baena. Augmented reality based navigation for computer assisted hip resurfacing: a proof of concept study. Ann. Biomed. Eng. 46:1-11, 2018.

${ }^{6}$ Singh, H., H. N. Modi, S. Ranjan, J. W. Dilley, D. Airantzis, G.-Z. Yang, A. Darzi, and D. R. Leff. Robotic surgery improves technical performance and enhances prefrontal activation during high temporal demand. Ann. Biomed. Eng. 46:1-16, 2018.

\section{BETHANY Rowson (D)}

Department of Biomedical Engineering and

Mechanics

Virginia Tech, Blacksburg, VA

Electronic mail: browson@vt.edu

Publisher's Note Springer Nature remains neutral with regard to jurisdictional claims in published maps and institutional affiliations. 